\title{
MULTI-FREQUENCY SYNTHESIS
}

\author{
J. E. CONWAY \\ Owens Valley Radio Observatory and Jet Propulsion Laboratory, \\ California Institute of Technology, Pasadena, CA 91125, U.S.A.
}

\begin{abstract}
A method of improving image fidelity by using observations at multiple frequencies is described. We discuss the power and possible limitations of the technique. Results of narrow frequency range observations with MERLIN and global VLBI are presented. We conclude with a consideration of the possible future impact of this technique.
\end{abstract}

\section{INTRODUCTION}

Aperture synthesis observations with sparse arrays usually have insufficient data to fully constrain their output images, giving errors which limit our astrophysical interpretation. Non-linear image reconstruction algorithms must be applied which effectively interpolate from the measured uv data to estimate unmeasured Fourier components. The larger the holes in the uv coverage, or the more complex the image, the larger the 'reconstruction errors' which result. In Multi-Frequency Synthesis observations are made at a number of frequencies $n_{f}$ over a $10 \%$ to $30 \%$ range. Changing frequency changes the spatial frequency of the Fourier components that we measure. With MFS the gaps in the uv coverage are much reduced (see Fig 1) and so we can obtain higher fidelity images (Conway, Cornwell and Wilkinson 1990, which we will refer to as $\mathrm{CCW})$.

The problem with this technique is that the source structure could potentially vary dramatically with frequency. But in continuum sources the spectrum at each pixel is fairly smooth, and the images at the different frequencies are highly correlated, it is these facts which allow us to contemplate using MFS. We find that the effects of frequency dependent structure are often below the thermal noise, as explained below, and in these cases we can obtain the full benefit of having $n_{f}$ times more data. Even when spectral effects exceed the thermal noise we find that they can usually be accounted for if we parameterize the image in terms of two unknowns at each pixel; the intensity at some average frequency and some form of frequency derivative. The use of MFS then doubles the number of unknowns for which have to solve but we 

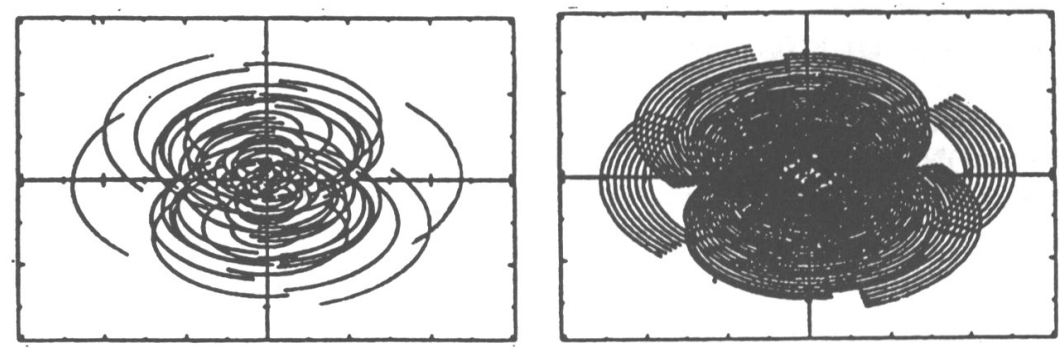

Fig. 1. Aperture coverages achievable with the full VIBA at declination $30^{\circ}$. Left: at a frequency of $1660 \mathrm{MHz}$. Right: Using MFS with 8 frequencies spread over $1350-1750 \mathrm{MHz}$.

have now $n$, more observables. If $n$, is greater than 2 the MFS image remains better constrained than the single frequency image.

\section{SPECTRAL EFFECTS}

We now consider the nature and size of the effects that arise on combining uv data at different frequencies. If all parts of a source had the same spectrum we could simply adjust the amplitudes at each frequency and then combine the data in the aperture plane, invert and deconvolve and there would be no spectral effects in the resulting image. Even when the spectrum varies across the source it is always worth removing some 'average' spectrum to reduce spectral effects. After rescaling the amplitude at each frequency by a factor $g(\nu)$ the data at frequency $\nu$ are the same as from a source of brightness distribution $I^{\prime}(x, y, \nu)=I(x, y, \nu) g(\nu)$. On combining all these data sets in the aperture plane and Fourier inverting the dirty map can be written as the following expansion

$$
I^{\prime}\left(x, y, \nu_{0}\right) * B_{0}+\left.\frac{d I^{\prime}(x, y, \nu)}{d f(\nu)}\right|_{\nu=\nu_{0}} * B_{1}+\left.\frac{1}{2} \frac{d^{2} I^{\prime}(x, y, \nu !}{d f^{2}(\nu)}\right|_{\nu=\nu_{0}} * B_{2} \ldots
$$

where the symbol * denotes convolution and $f(\nu)$ is some function of frequency. The derivatives are functions of spatial coordinates $x$ and $y$ and will be referred to as 'coefficient images'. The $B_{n}$ are generalised dirty beams which are the Fourier transforms of the MFS uv coverage with each uv point reighted according to its frequency of observation by $(f(\nu))^{n}$. In CCW we considered the common case where the spectrum at each pixel is, to good approximation, a power law with position dependent spectral index $\alpha(x, y)$ so that $I(x, y, \nu)=$ $I\left(x, y, \nu_{0}\right)\left(\nu / \nu_{0}\right)^{-\alpha(x, y)}$. We assume that the amplitudes have been corrected for an 'overall' spectral index $\alpha_{0}$ so that $g(\nu)=\left(\nu / \nu_{0}\right)^{\alpha_{0}}$ and so $I^{\prime}=$ 

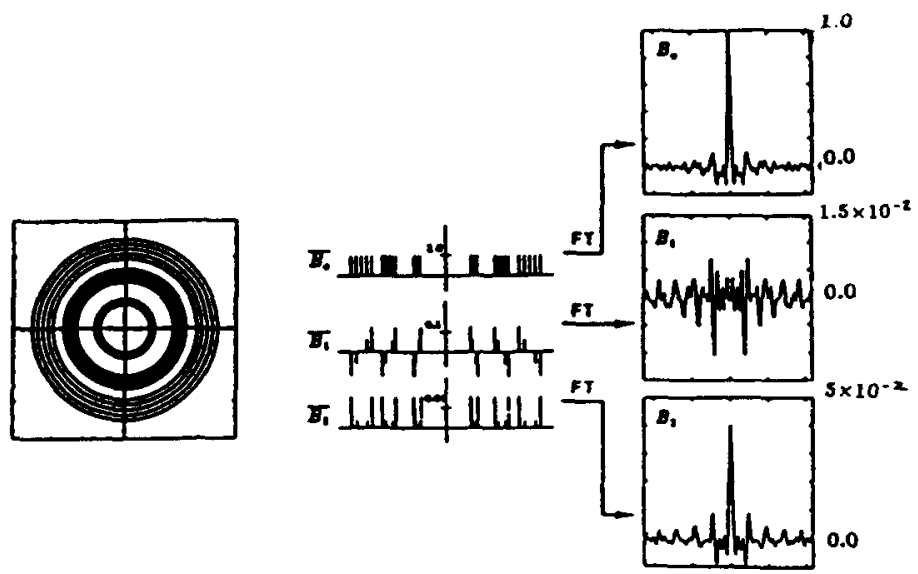

Fig. 2. Illustration of the nature of the spectral dirty beams. Left: A simple uv coverage formed from 3 antennas and 5 frequencies. Middle: A slice across the uv coverage showing the weightings corresponding to different order of spectral dirty beam. Right: Slices through the beams formed from Fourier transforming the weighted coverages.

$I\left(x, y, \nu_{0}\right)\left(\nu / \nu_{0}\right)^{-\alpha^{\prime}}$ where the residual spectral index $\alpha^{\prime}(x, y)=\alpha-\alpha_{0}$. We can expand in any form for $f(\nu)$ that we wish; for the case of power law spectra the choice $f(\nu)=\ln \left(\nu_{0} / \nu\right)$ is appropriate. The general expansion for the dirty map becomes

$$
I\left(x, y, \nu_{0}\right) * B_{0}+I(x, y) \alpha^{\prime}(x, y) * B_{1}+\frac{1}{2} I(x, y) \alpha^{\prime 2}(x, y) * B_{2} \ldots . .
$$

Fig 2, illustrates the nature of the spectral dirty beams for a simple example uv coverage. Note that there is a clear difference between the even and odd order dirty beams, the former have large central lobes whilst the latter have no such central peat. In CCW it was shown that that the peak sidelobes due to $B_{1}$ are of the order of $5 \times 10^{-3}$ for aperture coverages typical of VLBI arrays and a frequency range of about $25 \%$. We can often choose $\alpha_{0}$ such that $\alpha^{\prime}$ is about 0.1 or 0.2 in the brightest regions, so that the spectral errors can occur at levels of between $I / 1000$ and $I / 2000$, i.e. at dynamic ranges of a 1000 or so. For images with dynamic ranges below this level we can usually ignore the contributions due to spectral effects.

\section{DOUBLE DECONVOLITTION}

Even if spectral effects are important, the dirty map can usually, within the noise, be described by the first two terms of the Taylor expansion. In such situations we need an algorithm which solves for both the $I(x, y)$ and $I \alpha^{\prime}(x, y)$ coefficient images. Various approaches to this problem have been investigated (Conway 1988). One promising approach, Double Deconvolution (DD), is 
an iterative algorithm which alternately attempts to estimate the $I$ and $I \alpha^{\prime}$ distributions. It does this by convolving the map alternately with each of the two spectral dirty beams, $B_{0}$ and $B_{1}$. and then CLEANing the result with the appropriate dirty beam. The algorithm is explicitly based on the original description of CLEAN (Högbom 1974) as a beam recognition algorithm. For further details we refer the reader to CCW.

Figure 3 illustrates the effectiveness of the algorithm with simulated data. Fig $3 a$ shows the model image. Fig $3 b$ shows the reconstruction obtained on CLEANing conventional single frequency data; it is badly affected by reconstruction errors. Fig $3 \mathrm{c}$ shows the effect of simply combining the data sets reweighting, inverting and deconvolving. The ring-like 'spectral errors' are the contribution of the $I \alpha^{\prime} * B_{1}$ term in the expansion for the dirty map. Fig $3 \mathrm{~d}$ shows the result of applying two cycles of Double Deconvolution. The spectral errors are removed and a good reconstruction is achieved.

As in CLEAN, it can be shown that DD is guaranteed to converge to fit the data. In situations in which the number of independent observables is more than double the number of filled pixels in the image, convergence to fit the data should give an image that is unique and true within the noise. To be well conditioned with respect to noise the fractional frequency ranges must span the gaps in the uv coverage; in fact some overlap between different physical baselines at different frequencies will be required. Such situations are feasible for some practical implementations of MFS, giving rise to the promise of almost unique images in such cases. More often, however, despite MFS, there will still be fewer constraints than unknowns. However, a successful separation between the effects of $I$ and $I \alpha^{\prime}$ using DD is still possible, because to achieve separation the algorithm implicitly uses the a priori information that the $I$ and $I \alpha^{\prime}$ distributions are compact. Futhermore it can be shown (Conway 1988) that if we switch from CLEANing one distribution to CLEANing the other before the residuals are dominated by the sidelobe effects of the distribution for which we are not CLEANing then progress toward a correct separation continues. In practice this means we should switch our CLEANing when the sidelobe levels in regions away from the source stop decreasing.

\section{COMPLICATIONS - HIGHER ORDER TERMS}

The DD algorithm assumes the dirty map can be expressed as a two order expansion. At high dynamic range this assumption will break down as the higher order terms become significant with respect to the thermal noise.

Because the second order beam is very similar to to the zeroth order beam and has a central peak (see Fig 2), its main effect is to add a small bias to the output image. The more important sidelobes which might obscure weak detail are much smaller. We can rearrange the general expansion to show that the dirty map equals

$$
\left[I^{\prime}\left(x, y, \nu_{o}\right)+B_{2}(0) \cdot \frac{1}{2} \frac{d^{2} I^{\prime}(x, y, \nu)}{d f^{2}(\nu)}\right] * B_{0}+\frac{d I^{\prime}(x, y, \nu)}{d f(\nu)} * B_{1}+\frac{1}{2} \frac{d^{2} I^{\prime}(x, y, \nu)}{d f^{2}(\nu)} * B_{2}^{\prime}
$$

evaluated at $\nu=\nu_{0}$. In this expression $B_{2}^{\prime}=\left(B_{2}-B_{2}(0) . B_{0}\right)$ and $B_{2}(0)$ is the central value of the second order beam (which for a $25 \%$ frequency range 


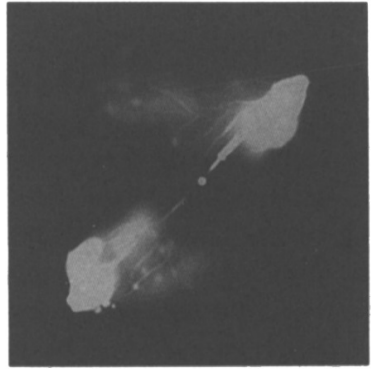

a)

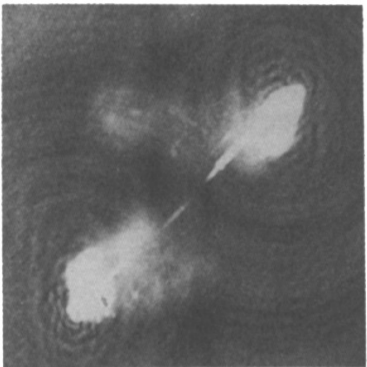

c)

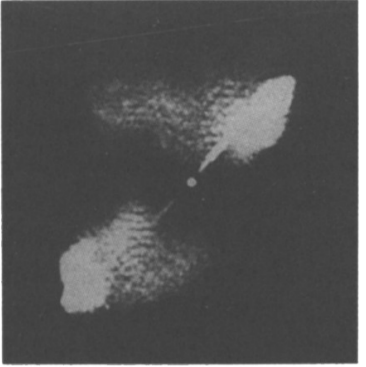

b)

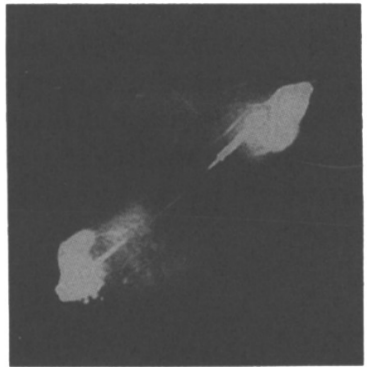

d)

Fig. 3. Applying Double Deconvolution to Simulated data. a) The model intensity distribution. b) The results of mapping single frequency data. c) The results of combining and deconvolving multi- frequency data. d) After applying two cycles of Double Deconvolution.

is about $1 / 200$ ). If we assume a two order expansion and apply DD we would obtain an I image biased by $B_{2}(0)$ times the second derivative of $I$ with respect to $f(\nu)$. In practice the bias on source will always be $<1 \%$. It will be strongly correlated with intensity variations and so is unlikely to mask weak detail or be astrophysically important. If we accept this bias the effective second order dirty beam equals $\left(B_{2}-B_{2}(0) \cdot B_{0}\right)$. This is very similar to the $B_{1}$ beam in having no central peak, only weak sidelobes. For a $25 \%$ frequency range these sidelobes are at the $5 \times 10^{-4}$ level (a figure which scales as the square of the frequency range).

The second order term that arises from a pure power law spectra $\left(I \alpha^{\prime 2} / 2\right)$ will be very small. For a hotspot with $\alpha^{\prime}=0.2$ the effective second order spectral sidelobes occurs at the $10^{-5}$ level. Even these small effects can potentially be removed by using the (slightly biased) estimates for $I$ and $I \alpha^{\prime}$ from DD to estimate and remove the $I \alpha^{\prime 2}$ terms. An iterative process should be able to estimate of I and $\alpha$ almost exactly (Cornwell 1984). More generally the spectra are affected by synchrotron self-absorption or electron aging which give second order derivatives which cannot be derived from the lower derivatives. One important case occurs in imaging core-dominated sources. Usually the unresolved core has a slightly curved spectrum. One way to reduce the second order effects in this case is to rescale the amplitude at each frequency by $g(\nu)=1 / S(\nu)$ where $S(\nu)$ is the total flux of the source at 
frequency $\nu$. This effectively removes the curvature in the spectrum of the brightest 'core' component and transfers it into the effective spectra of the much weaker secondary components, which will have much smaller second order spectral spectral errors. The most challenging situation for MFS occurs in $\mathrm{cm}$ wavelength global VLBI, in which the image often contains several spatially resolved self-absorbed components which peak at different frequencies. For the source NRAO140 the spectra of the multiple components within the jet have been determined (Marscher 1988). If MFS observations of this source with the VLBA plus Bonn were made in L-band with a $25 \%$ frequency range simulations show that the effective second order effects would be dominated by a single component which peaks near the observing band, giving peak second order sidelobes at $5 \times 10^{-4}$ of the peak intensity. Fortunately in this source and others significant curvature at the observing frequency is limited to only a small part of the jet. We could image each frequency separately and estimate the spectral curvature within that small region. After removing the effects of this spectral curvature from the MFS data we can obtain much higher dynamic range MFS images.

\section{COMPLICATIONS - SELF CALIBRATION}

Although by using $n_{f}$ frequencies we can make the uv coverage equivalent to that obtained by an array with $\sqrt{n_{f}}$ times as many telescopes it will not be equivalent in the number of phase closure constraints. If self-calibration is done at each frequency separately the ultimate fidelity of our MFS map may be limited by 'self-calibration errors' which depend on the single frequency aperture coverages. No matter how well MFS fills the plane there will only be a statistical improvement on combining the data sets.

One way to improve things is to use the output $I$ and $I \alpha^{\prime}$ from DD to generate model images at each of the frequencies, and then to use these model images in separate self-calibrations at each frequency. The corrected data could be used as input for a new DD cycle and so on iteratively. This method at least indirectly uses the information at all the frequencies to constrain the self-calibration solution. Much more can be done if the multifrequency data are collected simultaneously or almost simultaneously (by rapid frequency switching) at the different frequencies. We can use the fact that the antenna-based phase errors are highly correlated to constrain our self-calibration solutions. In principle we need only solve at each antenna for a delay term (phase $\propto \nu$ ) and at low frequencies an ionospheric term (phase $\propto 1 / \nu$ ). Determining the phase error at each antenna absolutely from the frequency dependence of phase is likely to be badly conditioned with respect to noise. More practically we might solve at each antenna for a phase error at an average frequency plus a linear gradient in frequency plus higher terms (if the ionospheric is significant). If we have only two unknowns for each of the $N$ antennas then the ratio of constraints (uv points) to unknowns (source visibilities plus antenna phase parameters) is approximately $n_{f} N /\left(n_{f} N+4\right)$; for high enough $n_{f}$ this ratio can exceed that for an 'equivalent coverage' single frequency array which is $\sqrt{n_{f}} N /\left(\sqrt{n_{f}} N+2\right)$. 


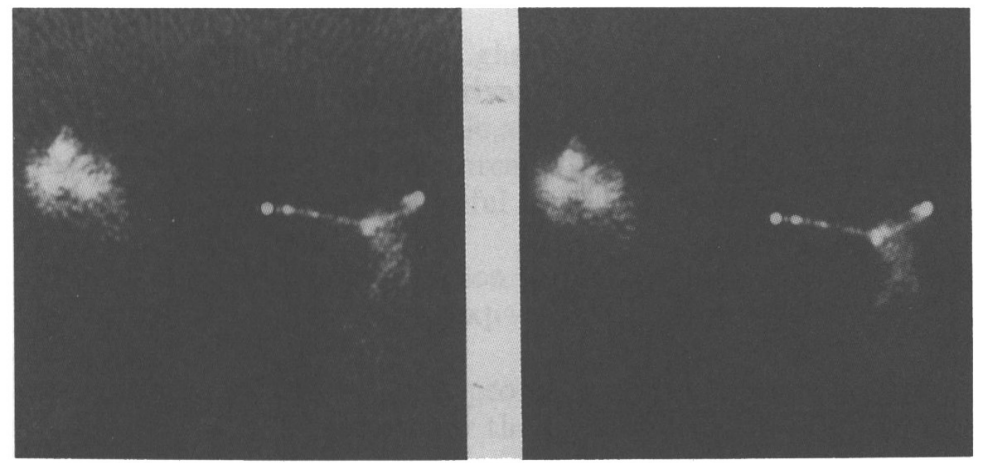

Fig. 4. Results of MFS mapping with MERLIN of the complex source $3 \mathrm{C} 179$ at L -band. Left: the best single frequency map. Right: MFS map made from five frequencies over $7 \%$.

\section{OBSERVATIONAL RESULTS}

We briefly describe the results of two observational tests of the MFS technique. In both cases the achieved frequency ranges were rather small $(<8 \%)$, and insufficient to reach across the gaps in the single frequency aperture coverage. Simulations suggest that in such cases the improvements in fidelity can be useful, but not as dramatic as that which can be seen by contrasting Fig $3 \mathrm{~b}$ and $3 \mathrm{~d}$. In both tests the fractional spectral dirty beam sidelobes and the required dynamic range were such that the expected spectral errors were below the noise level and there was no need to use the DD algorithm.

Observations of the complex high declination source $3 \mathrm{C} 179$ were made at 5 frequencies in L-band $(1612,1632,1662,1690$ and $1720 \mathrm{MHz})$ with the MERLIN array. Each of the frequencies was observed on a different day. The data at each frequency were separately mapped and self-calibrated. The amplitudes at each frequency were rescaled by a spectral index of 0.5 and then the data were combined in the uv plane together with a single snapshot of VLA A-array data. The resulting image after inversion and CLEANing is shown in Fig 4 together with the best single frequency image (at $1632 \mathrm{MHz}$ ). There are small but clear improvements, especially in the removal of the striping effect in parts of the western lobe.

In September 1989 global VLBI observations of the source 3C 84 were made at frequencies of $21.7,22.2$ and $23.5 \mathrm{GHz}$, again on separate days. This target and frequency band were chosen for the first MFS VLBI observations because even at low dynamic range (less than 100:1) the structure was too complex to be constrained by the available single frequency aperture coverages. To date the middle and higher frequency have been successfully fringe-fitted, self-calibrated, combined, inverted and CLEANed to give the provisional map of the inner few parsecs shown in Fig 5. On going from a single frequency to two frequencies the dynamic range of our image improved almost immediately by a factor of 3 . Processing of the MFS data is continuing. 

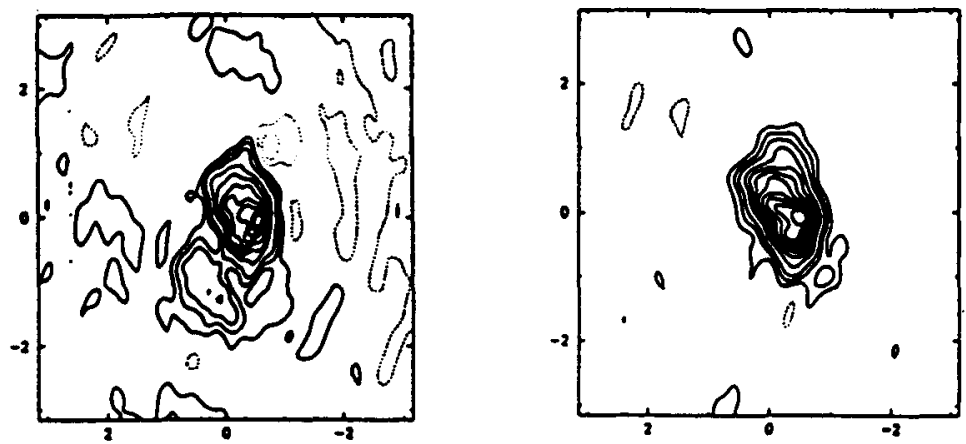

Fig. 5. Results of MFS mapping with Global VLBI of the nuclear region of $3 C 84$ at $K$-band. Left: the best single frequency map. Right: MFS map made from two frequencies separated by $5 \%$. Contour levels at $-2,2,5,10,20 \ldots 100 \%$. Restoring beam is 0.3 mas circular.

\section{FUTUREIMPACT}

The MFS technique has an important role to play in improving the fidelity of images produced from sparse arrays such as the VLBA/Global VLBI, MERLIN and the AT. With the advent of the VLBA the complexity of VLBI images will no doubt dramatically increase as high sensitivity systems are regularly used to observe some of the brightest sources. At the same time the number of antennas available will not be dramatically increasing. Fortunately the VLBA is in a good position to exploit MFS. Unlike most present VLBI antennas it can tune over a wide frequency range so that at most bands it can cross most of the gaps in the single frequency uv coverage. Furthermore it can observe up to 8 frequencies simultaneously over about $400 \mathrm{MHz}$ allowing multi-frequency self-calibration. More frequencies can be added by switching the LO frequency. The Australia Telescope is also well designed for MFS; full aperture coverage can normally only be achieved by moving the antennas, but by using MFS the speed and versatility of the instrument can be greatly improved.

This work has been supported in part by the National Science Foundation, grant number AST-8814554.

\section{REFERENCES}

Conway, J. E. 1988. PhD Thesis, University of Manchester.

Conway, J. E., Cornwell. T.J. and Wilkinson, P.N. 1990. M.S.R.A.S., 246, 490.

Cornwell, T. J. 1984. ILBA Memo No 324.

Högbom, J. A. 1974 . Astr. Ap. Suppl., 15, 417.

Marscher, A. P., 1988. Ap. J., 334, 552. 
Ray Norris: Have you considered using a two-parameter model to account for complex source spectra, allowing very wide bandwidths (e.g. octaves) to be used?

John Conway: Clearly for the very highest dynamic range or octave bandspread such a fit to the higher derivative coefficient images would be useful. I doubt however that any simple adaptation to Double Deconvolution could be made which could solve for three unknowns per pixel. A MEM based algorithm would probably be more useful for this problem.

David Murphy: An obvious application for Multi-Frequency Synthesis is space VLBI. What benefits could we expect to gain and what would be the limitations?

John Conway: In order to greatly improve fidelity it is important to cross the gaps in the uv-plane. The holes for the proposed space VLBI missions are (at least) octave sized, frequency ranges of $<30 \%$ may be useful, but will not give a really dramatic improvement. With octave frequency ranges we have all the problems of spectral curvature and higher derivatives. Multi-frequency modifying using synchrotron self-absorbed components may however be a good approach to deriving physical parameters and morphology from multifrequency uv data. 